\title{
LncRNA RUSC1-AS1 promotes the proliferation of hepatocellular carcinoma cells through modulating NOTCH signaling
}

\author{
Y. A. CHEN ${ }^{1, *}$, L. CHENG ${ }^{2, *}$, Y. ZHANG ${ }^{1}$, L. PENG ${ }^{1}$, H. G. YANG ${ }^{3, *}$ \\ ${ }^{1}$ Department of Oncology, Jimin Hospital, Shanghai, China; ${ }^{2}$ Echocardiography Lab Huashan Hospital, Fudan University Shanghai, Shanghai, \\ China; ${ }^{3}$ Department of Oncology, First People's Hospital of Suzhou City, Suzhou, Anhui, China \\ ${ }^{*}$ Correspondence: guangeng75601@163.com \\ ${ }^{*}$ Contributed equally to this work.
}

Received May 21, 2018 / Accepted July 22, 2019

\begin{abstract}
The long non-coding RNA (lncRNA) RUSC1-AS1 has been reported to be dysregulated in the progression of many cancers. Also, RUSC1-AS1 had been detected to be highly expressed in laryngeal squamous cell carcinoma and breast cancer cells, suggesting that RUSC1-AS1 may be a biomarker for cancers. However, the biological role and regulatory mechanism of RUSC1-AS1 in hepatocellular carcinoma (HCC) remain unknown. In this study, we found that RUSC1-AS1 was upregulated in HCC tissues and cells, and predicted unfavorable prognosis of HCC patients. The function assays including colony formation, EdU, TUNEL assay revealed that RUSC1-AS1 facilitated HCC cell proliferation and inhibited HCC cell apoptosis. Furthermore, mechanism assays including luciferase reporter assay and RIP assay demonstrated that RUSC1AS1 could directly bind to hsa-miR-7-5p. Besides, hsa-miR-7-5p targeted and negatively regulated NOTCH3 expression. Moreover, RUSC1-AS1 sponged hsa-miR-7-5p to upregulate NOTCH3 and to trigger the NOTCH signaling pathway. The rescue assays depicted that RUSC1-AS1 regulated HCC cell proliferation and apoptosis through modulating NOTCH signaling. In conclusion, lncRNA RUSC1-AS1 promoted the proliferation and reduced the apoptosis of HCC cells through activation of NOTCH signaling via hsa-miR-7-5p/NOTCH3 axis.
\end{abstract}

Key words: long non-coding RNA, RUSC1-AS1, hsa-miR-7-5p, NOTCH3, hepatocellular carcinoma

Hepatocellular carcinoma (HCC) is one of the most common tumors and is the third cause of cancer mortality worldwide [1-3]. The majority of HCC cases occurred in Asia and Africa [4]. HCC incidence has been on an increase, especially in men, in the last thirty years [5]. Also, the burden of HCC in medicine and the economy will have a drastic increase in the next decades on western populations according to epidemiologic evidence $[5,6]$. Although much scientific work and significant progress have been made in the basic cellular mechanism of HCC, the specific mechanisms underlying liver carcinogenesis still remain uncovered. Even more, the 5-year survival rates of HCC have not been improved in the last several years $[7,8]$. So, new diagnostic biomarkers and therapeutic strategies for improving the diagnosis and prognosis of HCC patients are in need. Then, the research on HCC is really essential.

Non-coding RNAs (ncRNAs) are generally classified into two groups according to the transcript size: small ncRNAs and long non-coding RNAs (lncRNAs) [9]. As a member of small ncRNAs, microRNAs (miRNAs) bind to the 3'-untrans- lated region (3'-UTR) of mRNAs, which controls mRNAs translation and degradation [10]. Significant evidence proves that miRNA is important in regulating cancer cell proliferation and apoptosis [11]. Moreover, recent studies on IncRNA have revealed the complex regulation mechanism of lncRNAs on genes [12]. Besides, it has been suggested that there are various types of lncRNA, such as cell type-, tissue type-, etc., which suggests that lncRNAs can play various functions in various diseases [13]. It has been proved that HCC tumorigenesis, metastasis, prognosis, and diagnosis are firmly related to dysregulation of lncRNAs including HULC, RERT, and HOTTIP/HOXA13 [14-16], which provides a new prospect for studying molecular mechanisms of HCC.

LncRNAs exert crucial roles in molecular signaling pathways, tethers, and decoys at the transcriptional, posttranscriptional, and translational level [17-19]. In recent studies, lncRNAs act as the role of competing endogenous RNAs (ceRNAs), namely miRNA sponges or antagonists [20]. Specifically speaking, lncRNAs downregulate the expression and activities of miRNAs and subsequently antagonize the 
repression of miRNAs on target genes at the post-transcriptional regulation level [21, 22].

LncRNA RUN and SH3 domain containing 1 antisense RNA 1 (RUSC1-AS1) had been detected to be highly expressed in laryngeal squamous cell carcinoma cells [23]. RUSC1-AS1 promotes breast cancer cell proliferation, suggesting RUSC1-AS1 as a biomarker for diagnosing breast cancer [24]. We aimed to explore the function and the potential ceRNA mechanism of RUSC1-AS1 in HCC cells. The results demonstrated that RUSC1-AS1 endogenously sponged hsa-miR-7-5p to elevate NOTCH3 expression, thus facilitating HCC cell proliferation and inhibiting HCC cell apoptosis. This study provided a new regulatory network of lncRNA-miRNA-mRNA in HCC.

\section{Materials and methods}

Tissue samples. 70 HCC tissues and adjacent healthy tissues were collected between June 2013 and July 2018, with approval from the Ethics Committee of Jimin Hospital. Before surgery, no patients received chemo- or radio-treatment. Each participant signed informed consent. Instantly after surgical resection, fresh tissues were frozen in liquid nitrogen and stored at $-80^{\circ} \mathrm{C}$.

Cell culture and treatment. HCC cell lines (Hep3b, HepG2, Huh7, HCCLM3) and human liver epithelial cells (THLE-3) were bought from the Chinese Academy of Sciences (Beijing, China) and cultured at $37^{\circ} \mathrm{C}$ in a humid $5 \% \mathrm{CO}_{2}$ incubator. Cell cultivation was carried out in DMEM (Invitrogen, Carlsbad, CA, USA) which was supplied with $10 \%$ fetal bovine serum (FBS; Invitrogen) and 1\% penicillin/ streptomycin (Sigma-Aldrich, Milan, Italy). HepG2 or Huh7 cells were treated with $3 \mathrm{mM}$ NOTCH1 agonist (VPA; Millipore, Darmstadt, Germany) for $48 \mathrm{~h}$ for activating NOTCH signaling.

Cell transfection. HepG2 and Huh7 cells were separately transfected with specific shRNAs against RUSC1-AS1 (sh-RUSC1-AS1\#1\#2; GenePharma, Shanghai, China) and the negative control (NC), pcDNA3.1/NOTCH3 and the empty pcDNA3.1 (GenePharma). The miR-7-5p mimics and NC mimics were obtained from GenePharma. Each plasmid was transfected into cells using Lipofectamine 2000 (Invitrogen).

qRT-PCR. Total RNA from cultured cells was extracted utilizing Trizol reagent (Invitrogen) and then reversetranscribed into cDNA according to a Reverse Transcription Kit (Invitrogen). qRT-PCR analysis was progressed using SYBR Green Premix PCR Master Mix (Roche, Mannheim, Germany) under an ABI HT9600 (Applied Biosystems, Foster City, CA, USA). Fold expression changes were calculated with $2^{-\Delta \Delta \mathrm{Ct}}$ method and GAPDH/U6 was seen as an endogenous reference.

Colony formation assay. HepG2 and Huh7 cells $\left(1 \times 10^{3}\right)$ were inoculated into 6-well plates. After cultivation for 2 weeks, cells were fixed with methanol (Sigma-Aldrich) and were then stained with crystal violet (Sigma-Aldrich). Finally, the visible colonies that included $\geq 50$ cells were counted manually.

EdU incorporation assay. Cells transfected with sh-RUSC1-AS1\#1\#2 or sh-NC were cultured with the EdU solution for $2 \mathrm{~h}$. Then, cells were fixed by using PBS (Solarbio, Beijing, China) containing 4\% paraformaldehyde (Sigma-Aldrich) and were maintained in 70\% ethanol (Sigma-Aldrich). Later, cells were stained by Cell-Light ${ }^{\mathrm{Tm}}$ EdU Apollo ${ }^{\circledR} 88$ In Vitro Imaging Kit (RioBio, Guangdong, China). Subsequently, cells were incubated with $100 \mu \mathrm{l}$ of $1 \times$ DAPI reaction solution followed by viability determination under a fluorescent microscope (Olympus, Tokyo, Japan).

TUNEL assay. Cell apoptosis was determined through the TUNEL staining assay using the In Situ Cell Death Detection Kit (Roche, Mannheim, Germany) based upon recommended guidelines. DAPI (Haoran Biotechnology, Shanghai, China) was applied for staining HepG2 and Huh7 cells, respectively. The fluorescence intensity was examined via an EVOS FL microscope (Olympus).

Apoptosis assay. HepG2 and Huh7 cells were cultured in 6-well plates and rinsed with PBS. After treatment, apoptosis cells were fixed in $70 \%$ ice-cooled ethanol for $2 \mathrm{~h}$ and resuspended in $300 \mu \mathrm{l}$ of binding buffer and then stained with Annexin V-FITC and propidium iodide in the dark for 10 min. After that, the cell apoptosis rate was detected via Flow Cytometer (Becton-Dickinson, New York, USA).

Western blot. Cell proteins were extracted by the utilization of RIPA lysis buffer (Beyotime, Beijing, China). BCA protein assay kit (Beyotime) was employed for measuring the protein concentration. Afterward, the proteins were separated by SDS-PAGE and then placed onto PVDF membranes (Invitrogen). The membranes were blocked with non-fat milk and then cultivated with primary antibodies for NOTCH3 (ab23426), Hes1 (ab71559), and GAPDH (ab8245) from Abcam (Cambridge, USA). Secondary antibodies were added for $1 \mathrm{~h}$. The proteins were visualized by the chemiluminescence detection system.

Subcellular fractionation. To determine the cellular localization of RUSC1-AS1, cytosolic and nuclear fractions were acquired using Nuclear/Cytoplasmic Isolation Kit (Norgenbiotek Corporation, Thorold, ON, Canada). RNAs were extracted from fractions and qRT-PCR analysis was applied for exploring the expression levels of RUSC1-AS1, U6 (nuclear control), and GAPDH (cytoplasmic control).

Luciferase reporter assay. The wild-type (Wt) and mutant (Mut) binding sites of miR-7-5p in the RUSC1-AS1 sequence or NOTCH3 3'UTR were inserted into the pmirGLO vector to construct plasmids, named RUSC1-AS1-Wt/Mut, NOTCH3-Wt/Mut. Then, the plasmids were co-transfected with miR-7-5p mimics or NC mimics into HepG2 and Huh7, severally. The luciferase activity was detected via Dual-Luciferase Reporter Assay System (Promega, MA, USA).

RIP assay. RIP experiment was carried out using a Magna RIP $^{\mathrm{ms}}$ RNA Binding Protein Immunoprecipitation Kit (Milli- 
pore). Briefly, cells were fixed in $1 \%$ formaldehyde and incubated overnight with anti-Ago2 (Millipore), anti-IgG (Millipore) as a negative control. qRT-PCR was performed for determining the expression levels of RUSC1-AS1, miR-7-5p in the precipitates.

RNA pull-down assay. The miR-7-5p-WT, miR-75p-MuT, and NC were biotin-labeled into Bio-miR-7-5p-WT, Bio-miR-7-5p-MuT, and Bio-NC. Furthermore, cell lysates were incubated with the biotinylated probe and M-280 streptavidin magnetic beads (Sigma-Aldrich). The relative expression of RUSC1-AS1 was analyzed by qRT-PCR afterward.

Statistical analysis. Statistical analysis was completed using SPSS 19.0 software (SPSS, USA) and data were expressed as means \pm SD. Student's t-test or one-way ANOVA was used to calculate the difference in groups. Overall survival was estimated by the Kaplan-Meier method, while gene correlation analysis was performed via Pearson's correlation analysis. A p-value $\leq 0.05$ had statistical significance. Besides, experiments were made independently three times.

\section{Results}

LncRNA RUSC1-AS1 is upregulated in HCC tissues and cells and predicts poor prognosis of HCC. For exploring the function of RUSC1-AS1 in HCC, first of all, qRT-PCR analyses were used to search the expression of RUSC1-AS1 in 70 paired of HCC tissues and non-tumor tissues (Figure 1A). The results depicted that RUSC1-AS1 was highly expressed in HCC tissues than in non-tumor tissues. Also, overall survival rates of HCC patients with high and low expression of RUSC1-AS1 were analyzed by the Kaplan-Meier method. Patients with high expression of RUSC1-AS1 had lower overall survival (Figure 1B). Meanwhile, we investigated the expression of RUSC1-AS1 in normal human liver cells (THLE-3) and HCC cells (HepG2, HUH7, Hep3b, and HCCLM3) by qRT-PCR analyses and found that RUSC1AS1 was upregulated in HCC cells (Figure 1C).

Secondly, qRT-PCR analyses were performed to verify the interference efficiency of RUSC1-AS1 (Figure 1D). Clone formation assays and EdU were implemented to assess the proliferation of HCC cells under different conditions of interfered RUSC1-AS1 (sh-RUSC1-AS1\#1 and sh-RUSC1AS1\#2). The results showed that interfered RUSC1-AS1 significantly decreased the proliferation of HCC cells (Figures 1E, 1F).

Thirdly, flow cytometry analysis and TUNEL were implemented to investigate the apoptosis affected by sh-RUSC1AS1\#1 and sh-RUSC1-AS1\#2. The results indicated that interfered RUSC1-AS1 enhanced the apoptosis of HCC cells (Figures 1G, 1H).

RUSC1-AS1 could bind to hsa-miR-7-5p. Then, a subcellular fraction assay was implemented to test the location of RUSC1-AS1 (Figure 2A), and RUSC1-AS1 was mostly located in the cytoplasm of HCC cells. To examine the ceRNA role of RUSC1-AS1, the downstream miRNAs for RUSC1-AS1 are in need to be explored. hsa-miR-7-5p was found response to silenced RUSC1-AS1 by qRT-PCR analyses (Figure 2B). RIP analyses were used to investigate the enrichment of miRNAs and RUSC1-AS1 in Ago-precipitated RISC (RNA induced silenced complex). The result proved that hsa-miR7-5 $p$ and RUSC1-AS1 were abundantly enriched in RISC in HepG2 cells (Figure 2C). Then, qRT-PCR analyses were used to investigate the expression of hsa-miR-7-5p in normal human liver cells and HCC cells (Figure 2D), and we found that hsa-miR-7-5p was lowly expressed in HCC cells.

Then, the bioinformatics tool predicted the binding site of RUSC1-AS1 and hsa-miR-7-5p; and we mutated the sequence of RUSC1-AS1 (Figure 2E). The overexpression efficiency of hsa-miR-7-5p was tested by qRT-PCR analyses (Figure 2F). Luciferase reporter assay was used to verify the binding between RUSC1-AS1 and hsa-miR-7-5p. Luciferase activity of wild RUSC1-AS1 was significantly decreased by hsa-miR-7-5p overexpression while that of mutant RUSC1AS1 was not impacted (Figure 2G). Meanwhile, RNA pulldown assay revealed that RUSC1-AS1 was significantly enriched in Bio-hsa-miR-7-5p-WT rather than Bio-hsamiR-7-5p-MuT, indicating that RUSC1-AS1 bound with hsa-miR-7-5p at predicted sites (Figure 2H). Also, a negative correlation was found between the expression of RUSC1-AS1 and hsa-miR-7-5p (Figure 2I).

NOTCH3 could bind to hsa-miR-7-5p and could be affected by hsa-miR-7-5p and RUSC1-AS1. Subsequently, the starBase tool was used to predict suitable mRNAs targeted by hsa-miR-7-5p, and 306 potential mRNAs have been found (Figure 3A). Then, we performed qRT-PCR for testing which mRNA could be affected by inhibited RUSC1-AS1 and overexpressed hsa-miR-7-5p (Figure 3B). The results revealed NOTCH3, ARL6IP1, CALM3, CKAP4, TNRC6A, NDUFA4, and ZC3H4 as the candidate mRNAs. Among them, only NOTCH3 is highly expressed in HCC cells (Figure 3C). The negative correlation between expression of hsa-miR-7-5p and NOTCH3 was revealed by Pearson analysis (Figure 3D). The putative binding site of wild/mutant NOTCH3 and hsa-miR-7-5p was displayed (Figure 3E). Then, the binding relationship between NOTCH3 and hsa-miR-7-5p was vindicated by luciferase reporter assay (Figure 3F). At last, the qRT-PCR analysis and western blot were conducted to test the influence of hsa-miR-7-5p and RUSC1-AS1 on NOTCH3. The results showed that the mRNA and protein level of NOTCH3 were downregulated by overexpression of hsa-miR-7-5p and downregulation of RUSC1-AS1 (Figures 3G, 3H).

Rescue assays were implemented to prove the interaction between NOTCH3 and RUSC1-AS1. After we have verified the axis of RUSC1-AS1/hsa-miR-7-5p/NOTCH3 in HCC cells, rescue assays were conducted to verify the axis. At first, the overexpression efficiency of $\mathrm{NOTCH} 3$ was investigated by qRT-PCR analysis (Figure 4A). Then, colony formation assay and $\mathrm{EdU}$ were implemented, and the groups were divided into sh-NC, sh-RUSC1-AS1, and sh-RUSC1- 


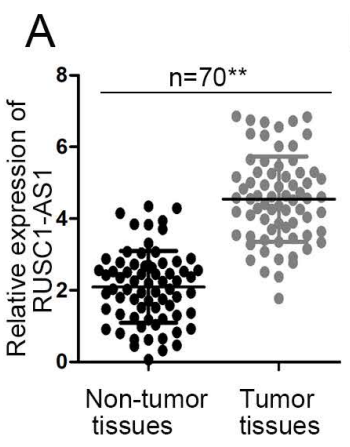

$\mathrm{E}$

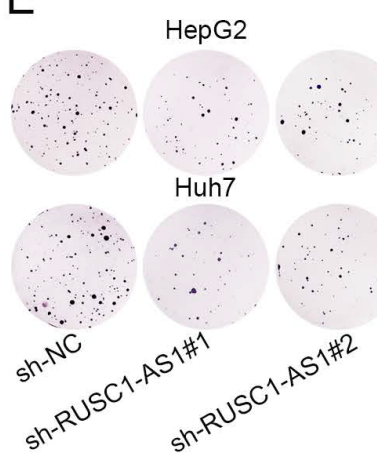

Huh7
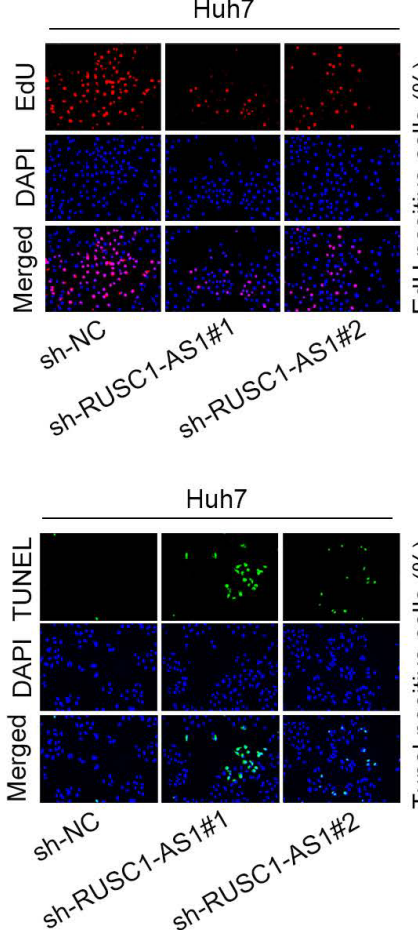
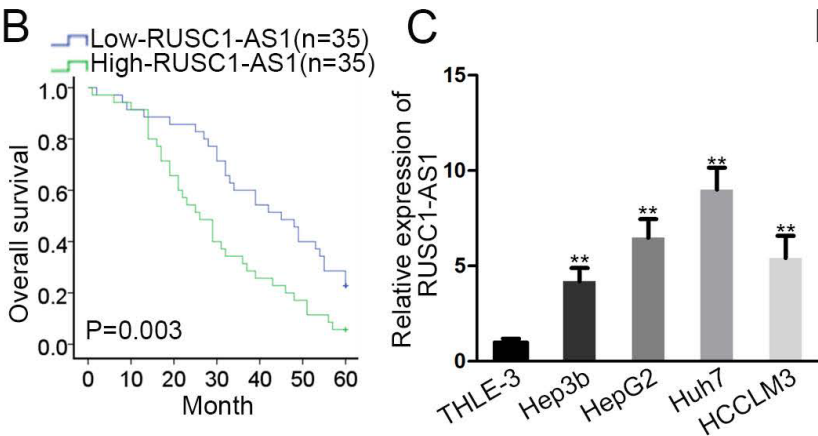

F

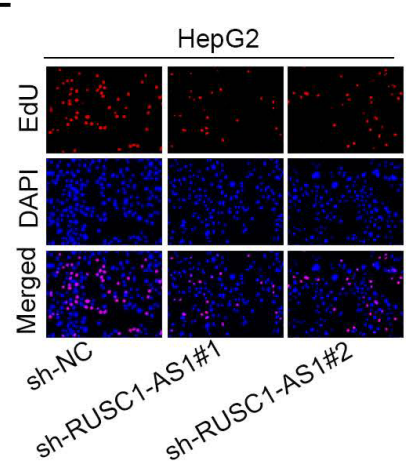

G
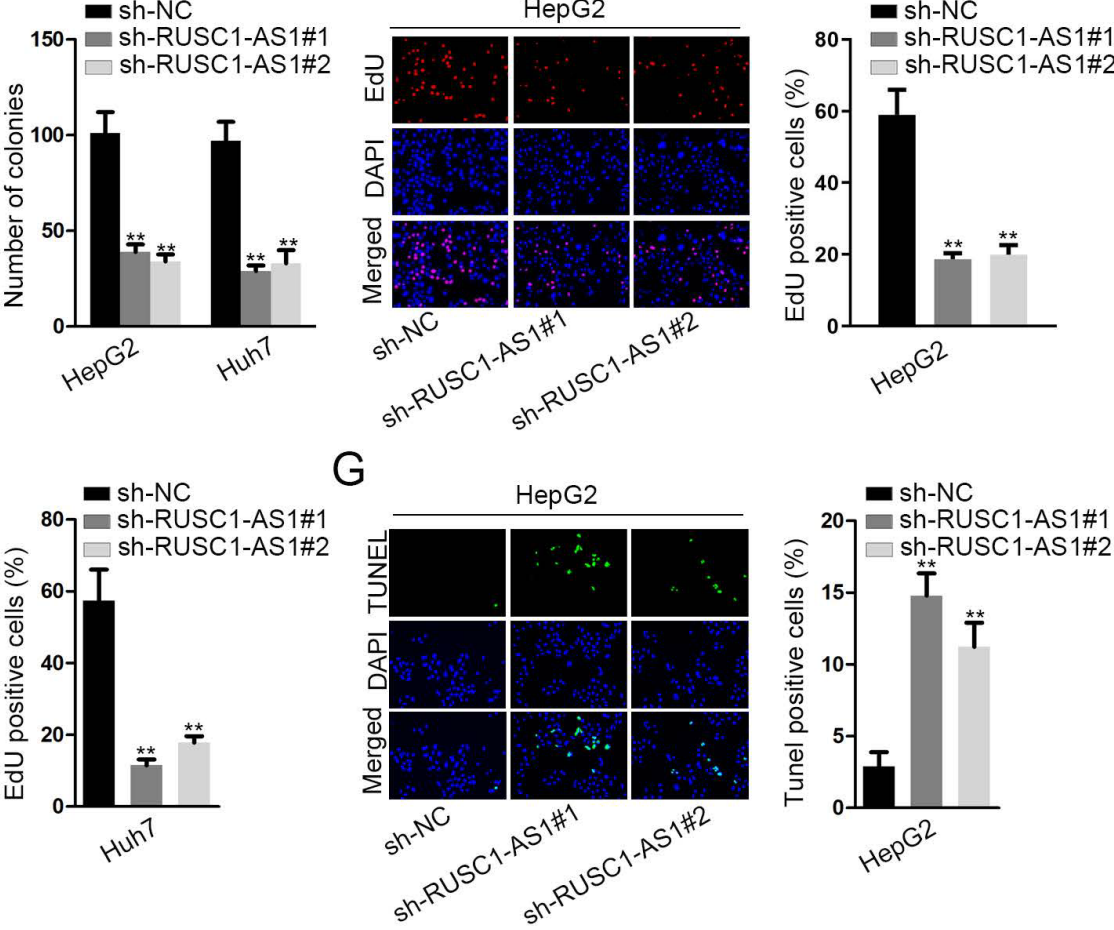

$\mathrm{H}$

ash-NC

${ }^{20} \square$ sh-RUSC1-AS1\#1

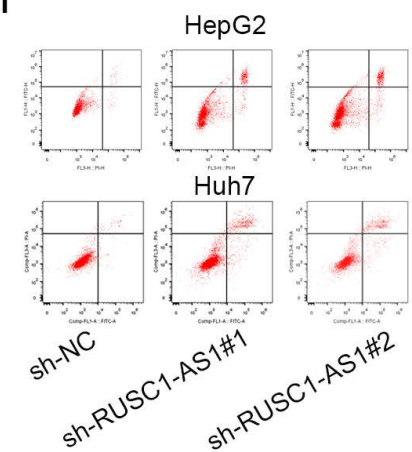

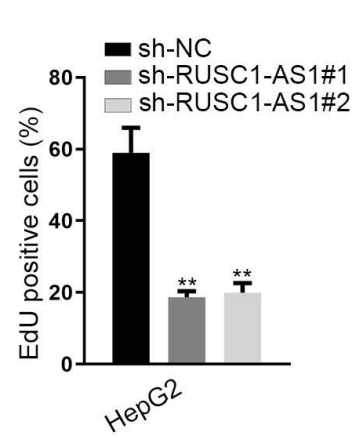
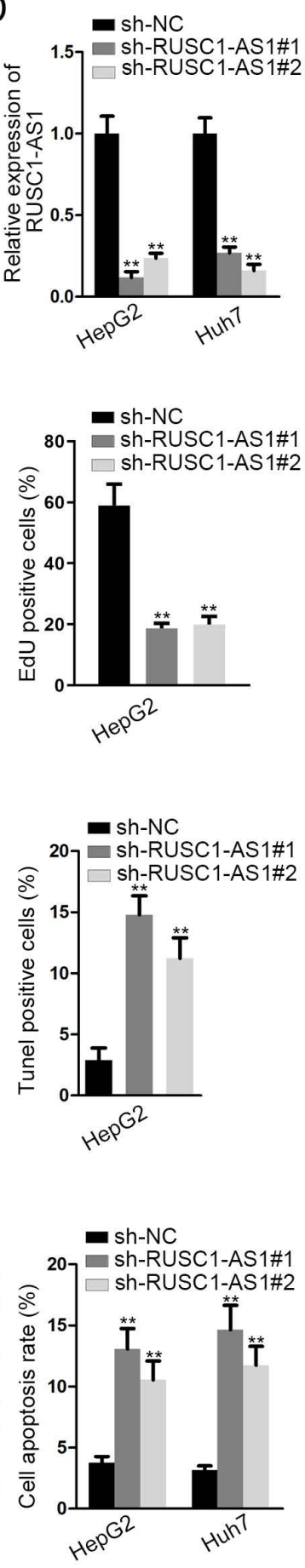

Figure 1. LncRNA RUSC1-AS1 is upregulated in HCC tissues and cells and predicts poor prognosis of HCC. A) qRT-PCR analyses were used to examine the expression of RUSC1-AS1 in HCC tissues and non-tumor tissues. B) Overall survival rates of HCC patients in high and low expression of RUSC1-AS1 were analyzed by the Kaplan-Meier method. C) The expression of RUSC1-AS1 in normal human liver cells (THLE-3) and HCC cells (HepG2, HUH7, Hep3b, and HCCLM3) was investigated by qRT-PCR analyses. D) qRT-PCR analyses were performed to test the interference efficiency of RUSC1-AS1. E, F) Clone formation assays and EdU were used to assess the proliferation of HCC cells under different conditions of interfered RUSC1-AS1 (sh-RUSC1-AS1\#1 and sh-RUSC1-AS1\#2). G, H) Flow cytometry analysis and TUNEL were implemented to investigate the apoptosis affected by sh-RUSC1-AS1\# 1 and sh-RUSC1-AS1\#2. ${ }^{\star *} \mathrm{p}<0.01$ 

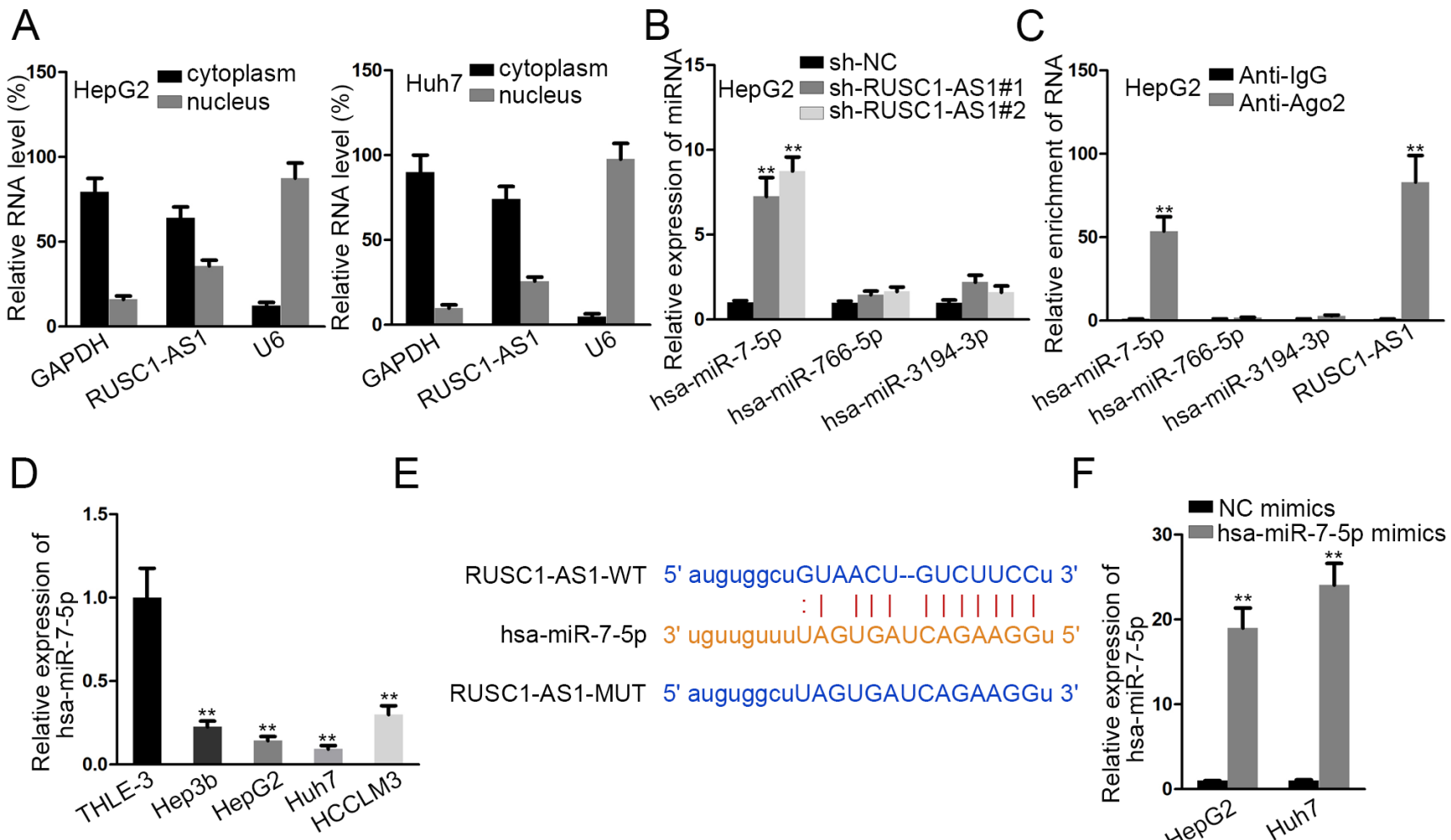

E

$\mathrm{F}$

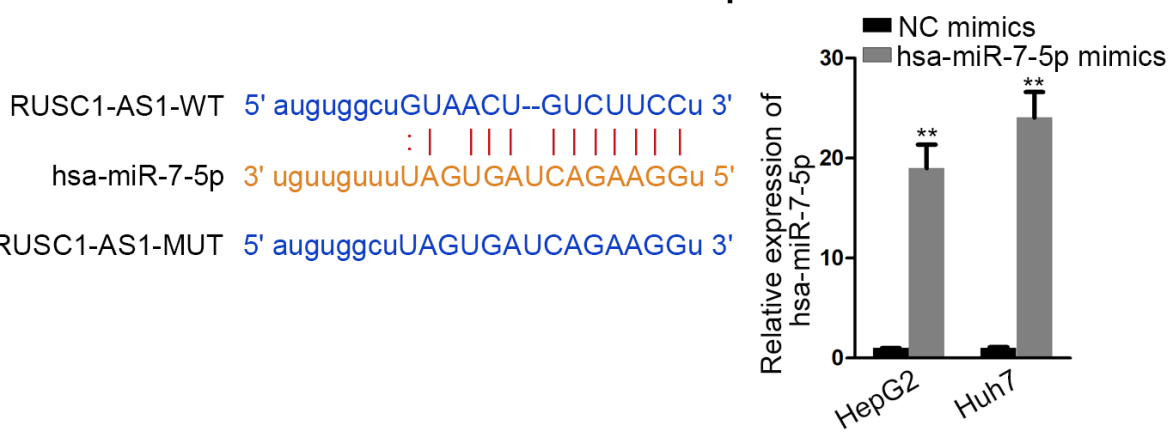

G

$\mathrm{H}$
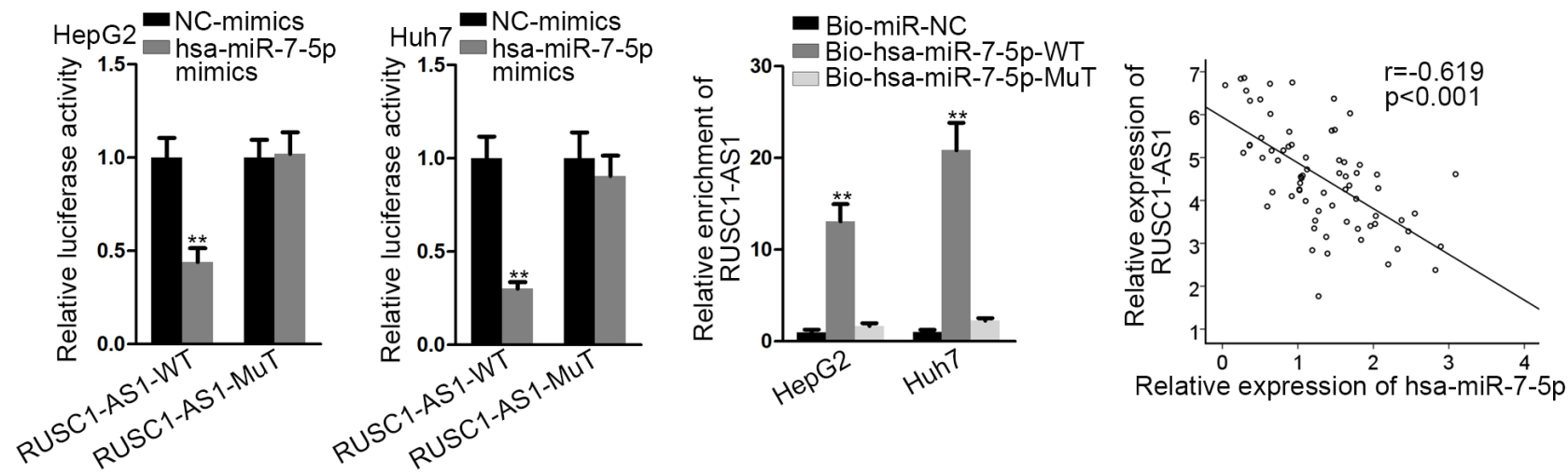

Figure 2. RUSC1-AS1 could bind to hsa-miR-7-5p. A) A subcellular fraction assay was implemented to test the location of RUSC1-AS1. B) qRT-PCR analysis was made to detect the expression change of miRNAs caused by inhibited RUSC1-AS1. C) RIP analyses were used to investigate the enrichment of miRNAs and RUSC1-AS1. D) qRT-PCR analyses were used to investigate the expression of hsa-miR-7-5p in normal human liver cells (THLE-3) and HCC cells (HepG2, HUH7, Hep3b, and HCCLM3). E) Bioinformatics presented the binding site of RUSC1-AS1 and hsa-miR-7-5p. F) The overexpression efficiency of hsa-miR-7-5p was investigated by qRT-PCR analyses. G) Luciferase reporter assay was used to examine the binding between RUSC1AS1 and hsa-miR-7-5p. H) RNA pull-down assay was performed to investigate the binding relationship between RUSC1-AS1 and hsa-miR-7-5p. I) A negative correlation was found between RUSC1-AS1 and hsa-miR-7-5p by Pearson analysis. ${ }^{* *}$ p $<0.01,{ }^{* * *} \mathrm{p}<0.001$

AS1+pcDNA3.1/NOTCH3. The results showed that the decreased proliferation of HCC cells caused by inhibited RUSC1-AS1 was rescued by overexpressed NOTCH3 (Figures 4B, 4C). Also, TUNEL showed that increased apoptosis of HCC cells caused by suppressed RUSC1-AS1 was offset by overexpressed NOTCH3 (Figure 4D). There- fore, the essential role of NOTCH3 in RUSC1-AS1-mediated cell functions was proved.

RUSC1-AS1 modulated the NOTCH signaling. In this section, we sought to examine whether RUSC1-AS1 modulated NOTCH signaling. Western blot was used to examine the impact of interfered RUSC1-AS1 on NOTCH 

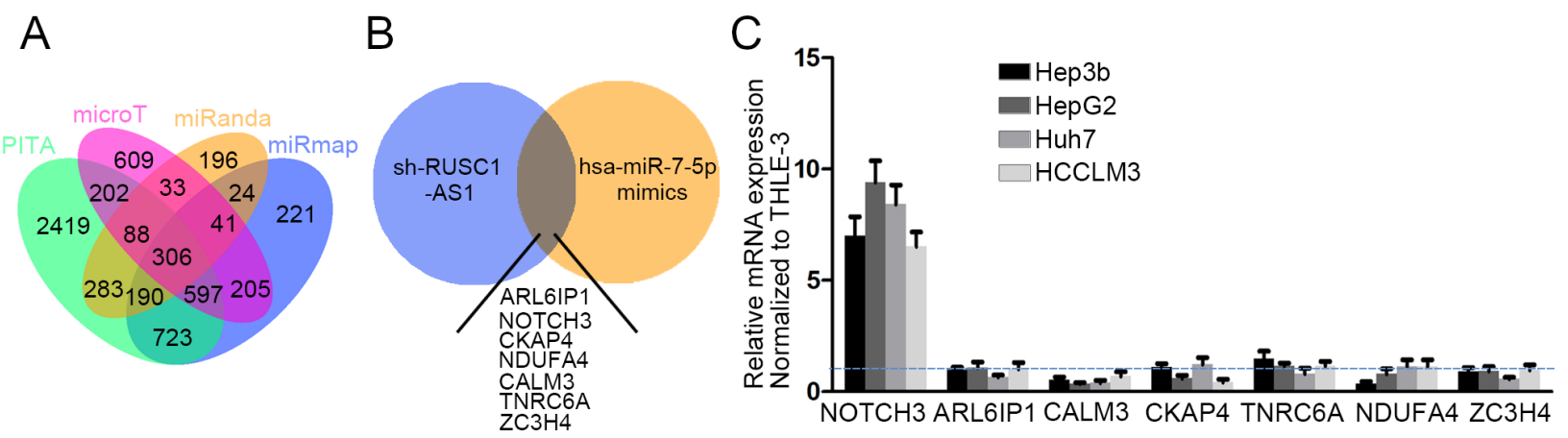

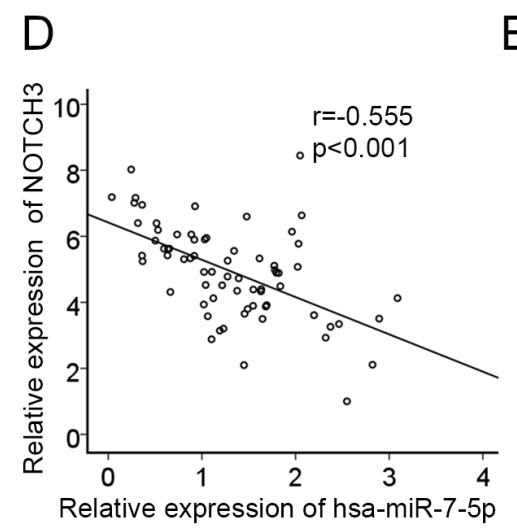

G

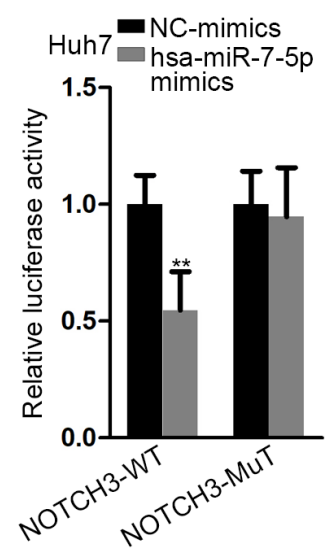

$E$

NOTCH3-WT Target: 5' cagcCCAGgGCUUCAGUCUUCCu 3' hsa-miR-7-5p miRNA: 3' uguuGUUUUAGUGAUCAGAAGGu 5' NOTCH3-MUT Target: 5' cagcGUUUUAGUGAUCAGAAGGu 3'

F

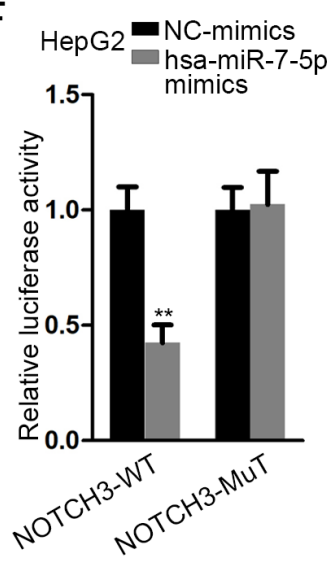

$\mathrm{H}$
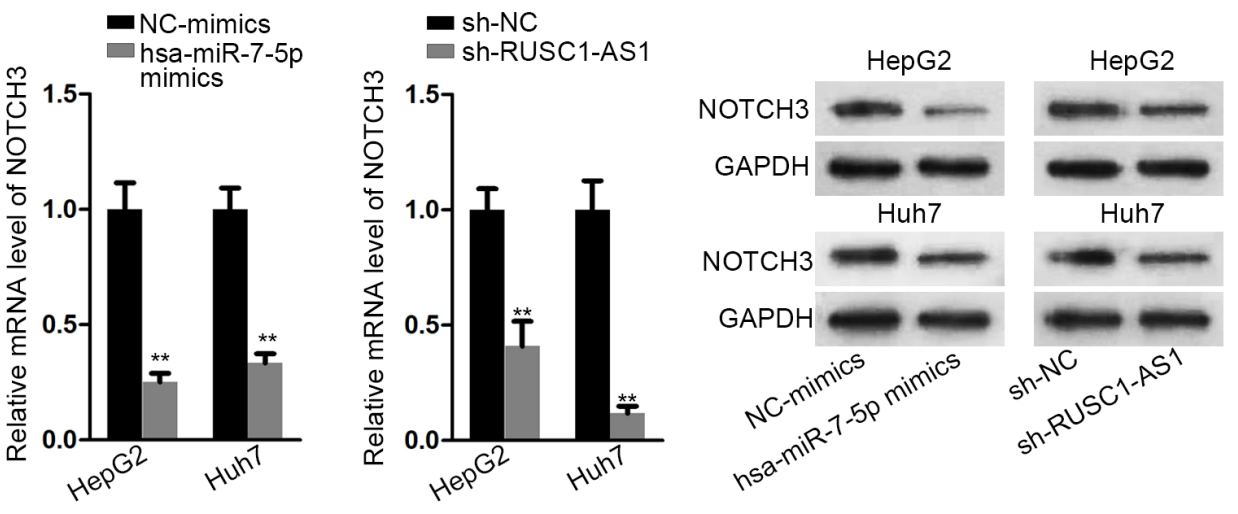

Figure 3. NOTCH3 could bind to hsa-miR-7-5p and could be affected by hsa-miR-7-5p and RUSC1-AS1. A) StarBase was used to predict suitable mRNA and 306 mRNAs have been found. B) qRT-PCR was made for testing which mRNA could be affected by inhibited RUSC1-AS1 and overexpressed hsa-miR-7-5p. C) The expressions of 7 mRNAs were investigated by qRT-PCR analysis. D) A negative correlation was found between hsa-miR-7-5p and NOTCH3. E) Bioinformatics presented the binding site of NOTCH3 and hsa-miR-7-5p. F) Binding relationship between NOTCH3 and hsa-miR-7-5p was vindicated by Luciferase reporter assay. G, H) qRT-PCR analyses and western blot was conducted to test the expression of NOTCH3 by overexpressed hsa-miR-7-5p and interfered RUSC1-AS1. ${ }^{* *} \mathrm{p}<0.01,{ }^{* * *} \mathrm{p}<0.001$

signaling (Figure 5A). The results indicated that the protein levels of Notch1 and Hes1 were decreased by suppressed RUSC1-AS1. Also, rescue assays were conducted to examine whether RUSC1-AS1 exerted its function via NOTCH signaling. We used NOTCH signaling pathway activator, VPA (valproic acid) [25], in the rescue assays. The results showed that decreased proliferation caused by inhibited RUSC1-AS1 was regained by VPA (Figures 5B, 5C). Also, from the results of TUNEL, increased apoptosis caused by inhibited RUSC1AS1 was offset by VPA (Figure 5D).

Above all, we concluded that RUSC1-AS1 acted as an oncogene in $\mathrm{HCC}$ through modulating $\mathrm{NOTCH}$ signaling. 


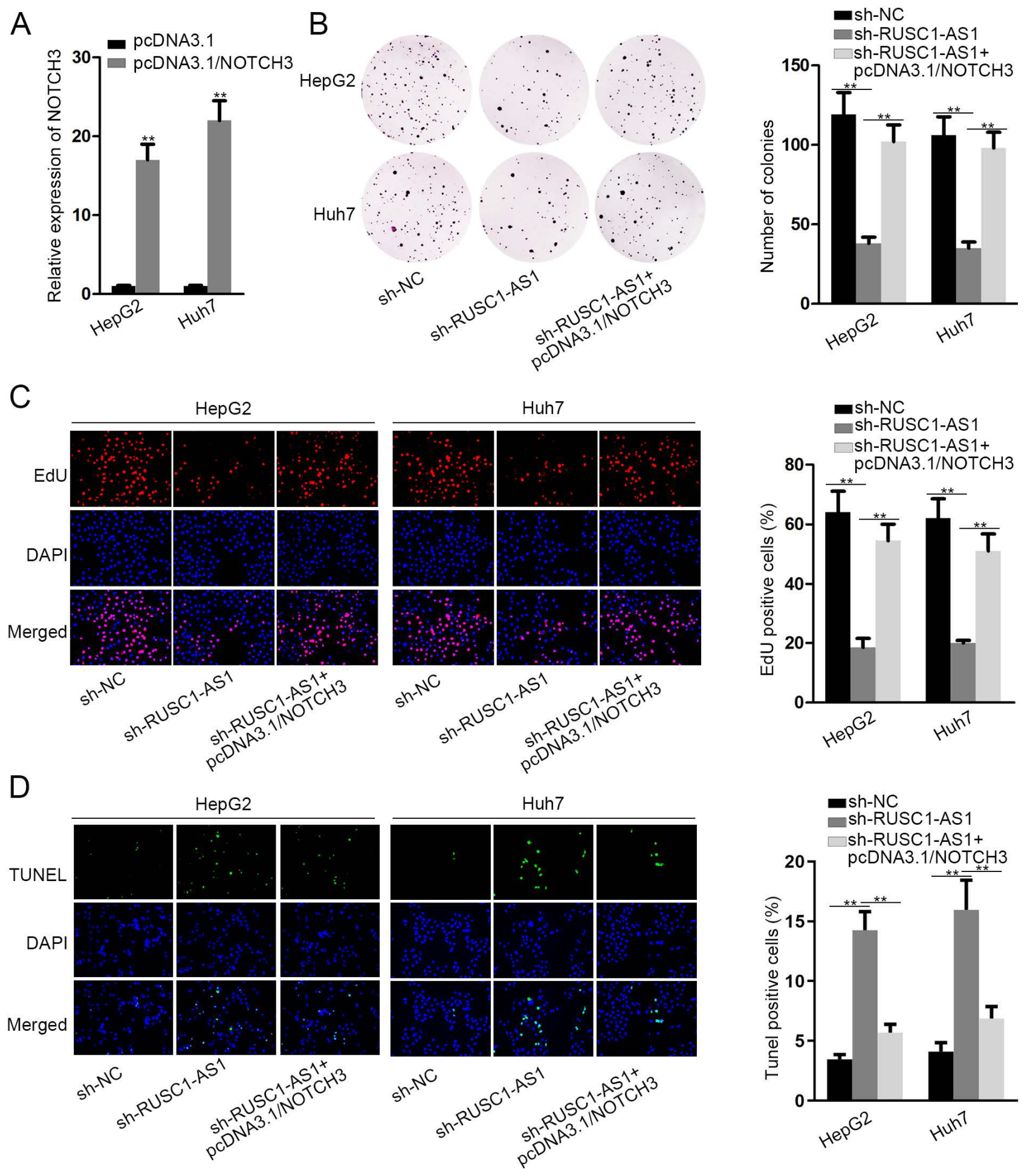

Figure 4. Rescue assays were implemented to prove the interaction in NOTCH3, hsa-miR-7-5p, and RUSC1-AS1. A) The overexpression efficiency of NOTCH3 was investigated by the QRT-PCR analysis. B, C) Colony formation assay and EdU were implemented to investigate the proliferation of HCC cells. D) TUNEL was used to investigate cell apoptosis situation. ${ }^{* *} \mathrm{p}<0.01$ 


\section{Discussion}

LncRNAs are a group of transcribed RNAs with more than 200 nucleotides in length and modulate genes in several aspects, such as in epigenetic aspects, transcriptional aspects, and post-transcriptional aspects [26]. More and more evidence indicates that different lncRNAs are obviously aberrantly expressed in cancers. These aberrantly-expressed lncRNAs are related to tumorigenesis, metastasis, and so on [27]. Therefore, we need to dedicate more efforts to explore the different functions of lncRNAs in cancers. In this study, we verified the overexpression of lncRNA RUSC1-AS1 in HCC tissues and cells. The results from function assays demonstrated that inter-
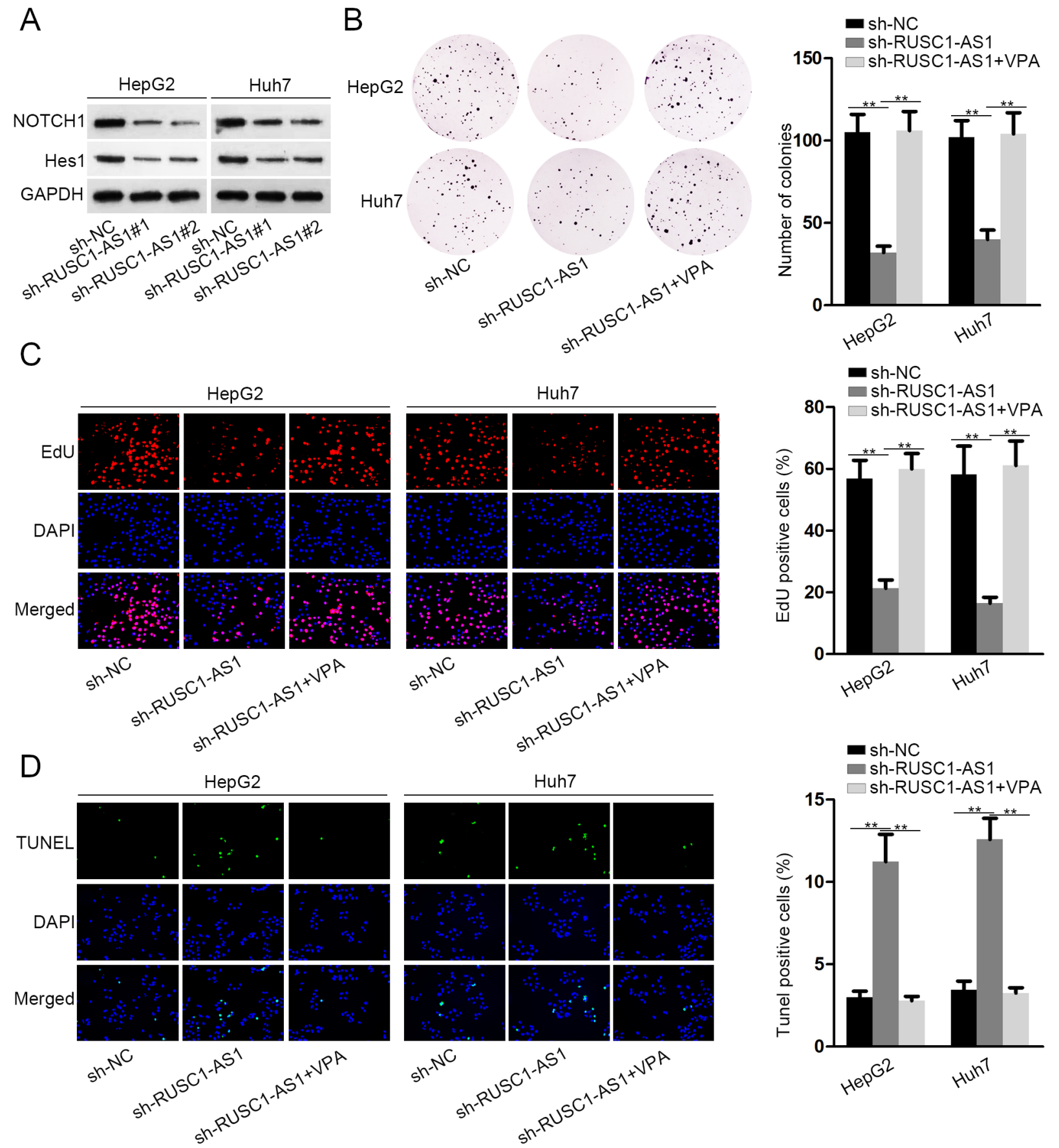

Figure 5. RUSC1-AS1 modulated NOTCH signaling. A) Western blot was used to test the impact of interfered RUSC1-AS1 on NOTCH signaling. B-D) Rescue assays were conducted by colony formation assay, EdU, and TUNEL to prove that RUSC1-AS1 exerted effects via NOTCH signaling. ${ }^{\star *}$ p $<0.01$ 
fered RUSC1-AS1 significantly decreased proliferation and increased apoptosis of HCC cells.

Meanwhile, miRNAs have also been researched as key players in human cancers, and they serve as oncogenes and suppressor genes in cancers. More importantly, recent research proved that lncRNA can play the role of miRNA sponge. LncRNAs interact with miRNAs and regulate the expression of mRNAs which were repressed by miRNAs $[19,21]$. For one example, lncRNA CHRF has the function of acting as an endogenous sponge of miR-489. Binding to miR-489, CHRF can modulate the miR-489 target gene, Myd88 [28]. For another example, acting as a ceRNA, IncRNA HOTAIR can effectively regulate the expression of miR-331-3p and then modulate the expression of HER2 in gastric cancer [29]. The ceRNA model in HCC was also widely reported. LncRNA OIP5-AS1 endogenously sponges miR-363-3p to upregulate SOX4, thus contributing to HCC progression [30]. LncRNA DUXAP8 facilitates HCC cell proliferation, migration, and invasion via modulation on the miR-422a/PDK2 axis [31]. In the present study, we identified that RUSC1-AS1 could bind with hsa-miR7-5p. miR-7-5p was reported to suppress tumor metastasis in non-small cell lung cancer by targeting NOVA2 [32]. miR-7-5p is sponged by CASC 21 and targets YAP1 in colorectal cancer [33].

Further, NOTCH3 was verified to be targeted and negatively regulated by miR-7-5p. RUSC1-AS1 served as a ceRNA against miR-7-5p to upregulate NOTCH3 and activate the NOTCH pathway. Subsequently, we explored whether RUSC1-AS1 exerted its pro-proliferation and anti-apoptosis effects via regulation of the $\mathrm{NOTCH}$ pathway. The rescue assays revealed that the agonist of the NOTCH pathway, VPA, completely rescued the effects of silenced RUSC1-AS1. NOTCH pathway is a common oncogenic pathway. LncRNA NALT1 contributes to cell invasion and migration in gastric cancer via the NOTCH signaling pathway [34]. LncRNA LBX2-AS1 promotes cell proliferation and migration through the Notch signaling in non-small cell lung cancer [35]. LncRNA SNHG7 interacts with miR-34a to contribute to tumorigenesis and progression of breast cancer through the Notch-1 pathway [36].

In summary, this study provides a new regulatory network of RUSC1-AS1/hsa-miR-7-5p/NOTCH3. Our present work indicated that RUSC1-AS1/hsa-miR-7-5p/NOTCH3 axis could modulate the proliferation and apoptosis of HCC cells through activation of the NOTCH signaling pathway.

\section{References}

[1] STACY S, HYDER O, COSGROVE D, HERMAN JM, KAMEL I et al. Patterns of consultation and treatment of patients with hepatocellular carcinoma presenting to a large academic medical center in the US. J Gastrointest Surg 2013; 17: 1600-1608. https://doi.org/10.1007/s11605-013-2253-y
[2] HARTKE J, JOHNSON M, GHABRIL M. The diagnosis and treatment of hepatocellular carcinoma. Semin Diagn Pathol 2017; 34: 153-159. https://doi.org/10.1053/j.semdp.2016.12.011

[3] KELLY D, SHARIF K, BROWN RM, MORLAND B. Hepatocellular carcinoma in children. Clin Liver Dis 2015; 19: 433-447. https://doi.org/10.1016/j.cld.2015.01.010

[4] SARTORIUS K, SARTORIUS B, ALDOUS C, GOVENDER PS, MADIBA TE. Global and country underestimation of hepatocellular carcinoma (HCC) in 2012 and its implications. Cancer Epidemiol 2015; 39(3): 284-90. https://doi. org/10.1016/j.canep.2015.04.006

[5] DHANASEKARAN R, LIMAYE A, CABRERA R. Hepatocellular carcinoma: current trends in worldwide epidemiology, risk factors, diagnosis, and therapeutics. Hepat Med 2012; 4: 19-37. https://doi.org/10.2147/HMER.S16316

[6] CLARK T, MAXIMIN S, MEIER J, POKHAREL S, BHARGAVA P. Hepatocellular Carcinoma: Review of Epidemiology, Screening, Imaging Diagnosis, Response Assessment, and Treatment. Curr Probl Diagn Radiol 2015; 44: 479-486. https://doi.org/10.1067/j.cpradiol.2015.04.004

[7] COLECCHIA A, SCHIUMERINI R, CUCCHETTI A, CESCON M, TADDIA $M$ et al. Prognostic factors for hepatocellular carcinoma recurrence. World J Gastroenterol 2014; 20: 5935-5950. https://doi.org/10.3748/wjg.v20. i20.5935

[8] LEE SC, TAN HT, CHUNG MC. Prognostic biomarkers for prediction of recurrence of hepatocellular carcinoma: current status and future prospects. World J Gastroenterol 2014; 20: 3112-3124. https://doi.org/10.3748/wjg.v20. i12.3112

[9] CHEN J, XUE Y. Emerging roles of non-coding RNAs in epigenetic regulation. Sci China Life Sci 2016; 59: 227-235. https://doi.org/10.1007/s11427-016-5010-0

[10] BARTEL DP. MicroRNAs: target recognition and regulatory functions. Cell 2009; 136: 215-233. https://doi.org/10.1016/j. cell.2009.01.002

[11] Di LEVA G, GAROFAlO M, CROCE CM. MicroRNAs in cancer. Annu Rev Pathol 2014; 9: 287-314. https://doi. org/10.1146/annurev-pathol-012513-104715

[12] AKHADE VS, PAL D, KANDURI C. Long Noncoding RNA: Genome Organization and Mechanism of Action. Adv Exp Med Biol 2017; 1008: 47-74. https://doi.org/10.1007/978981-10-5203-3_2

[13] TAY Y, RINN J, PANDOLFI PP. The multilayered complexity of ceRNA crosstalk and competition. Nature 2014; 505: 344-352. https://doi.org/10.1038/nature12986

[14] DU Y, KONG G, YOU X, ZHANG S, ZHANG T et al. Elevation of highly up-regulated in liver cancer (HULC) by hepatitis $\mathrm{B}$ virus $\mathrm{X}$ protein promotes hepatoma cell proliferation via down-regulating p18. J Biol Chem 2012; 287: 26302-26311. https://doi.org/10.1074/jbc.M112.342113

[15] ZHU Z, GAO X, HE Y, ZHAO H, YU Q et al. An insertion/ deletion polymorphism within RERT-lncRNA modulates hepatocellular carcinoma risk. Cancer Res 2012; 72: 6163 6172. https://doi.org/10.1158/0008-5472.CAN-12-0010 
[16] QUAGLIATA L, MATTER MS, PISCUOGLIO S, ARABI L, RUIZ C et al. Long noncoding RNA HOTTIP/HOXA13 expression is associated with disease progression and predicts outcome in hepatocellular carcinoma patients. Hepatology 2014; 59: 911-923. https://doi.org/10.1002/hep.26740

[17] EADES G, ZHANG YS, LI QL, XIA JX, YAO Y et al. Long non-coding RNAs in stem cells and cancer. World J Clin Oncol 2014; 5: 134-141. https://doi.org/10.5306/wjco.v5.i2.134

[18] QIU MT, HU JW, YIN R, XU L. Long noncoding RNA: an emerging paradigm of cancer research. Tumour Biol 2013; 34: 613-620. https://doi.org/10.1007/s13277-013-0658-6

[19] WANG K, LONG B, ZHOU LY, LIU F, ZHOU QY et al. CARL lncRNA inhibits anoxia-induced mitochondrial fission and apoptosis in cardiomyocytes by impairing miR539-dependent PHB2 downregulation. Nat Commun 2014; 5: 3596. https://doi.org/10.1038/ncomms4596

[20] QI X, ZHANG DH, WU N, XIAO JH, WANG X et al. ceRNA in cancer: possible functions and clinical implications. J Med Genet 2015; 52: 710-718. https://doi.org/10.1136/ jmedgenet-2015-103334

[21] CESANA M, CACCHIARELLI D, LEGNINI I, SANTINI T, STHANDIER O et al. A long noncoding RNA controls muscle differentiation by functioning as a competing endogenous RNA. Cell 2011; 147: 358-369. https://doi.org/10.1016/j. cell.2011.09.028

[22] KALLEN AN, ZHOU XB, XU J, QIAO C, MA J et al. The imprinted H19 lncRNA antagonizes let-7 microRNAs. Mol Cell 2013; 52: 101-112. https://doi.org/10.1016/j.molcel.2013.08.027

[23] HUI L, WANG J, ZHANG J, LONG J. lncRNA TMEM51AS1 and RUSC1-AS1 function as ceRNAs for induction of laryngeal squamous cell carcinoma and prediction of prognosis. PeerJ 2019; 7: e7456. https://doi.org/10.7717/ peerj.7456

[24] HU CC, LIANG YW, HU JL, LIU LF, LIANG JW et al. LncRNA RUSC1-AS1 promotes the proliferation of breast cancer cells by epigenetic silence of KLF2 and CDKN1A. Eur Rev Med Pharmacol Sci 2019; 23: 6602-6611. https://doi. org/10.26355/eurrev_201908_18548

[25] GREENBLATT DY, CAYO MA, ADLER JT, NING L, HAYMART MR et al. Valproic acid activates Notch1 signaling and induces apoptosis in medullary thyroid cancer cells. Ann Surg 2008; 247: 1036-1040. https://doi.org/10.1097/ SLA.0b013e3181758d0e

[26] PARASKEVOPOULOU MD, HATZIGEORGIOU AG. Analyzing MiRNA-LncRNA Interactions. Methods Mol Biol 2016; 1402: 271-286. https://doi.org/10.1007/978-1-49393378-5_21
[27] HE Y, MENG XM, HUANG C, WU BM, ZHANG L et al. Long noncoding RNAs: Novel insights into hepatocellular carcinoma. Cancer Lett 2014; 344: 20-27. https://doi. org/10.1016/j.canlet.2013.10.021

[28] WANG K, LIU F, ZHOU LY, LONG B, YUAN SM et al. The long noncoding RNA CHRF regulates cardiac hypertrophy by targeting miR-489. Circ Res 2014; 114: 1377-1388. https://doi.org/10.1161/CIRCRESAHA.114.302476

[29] WANG J, LIU X, WU H, NI P, GU Z et al. CREB up-regulates long non-coding RNA, HULC expression through interaction with microRNA-372 in liver cancer. Nucleic Acids Res 2010; 38: 5366-5383. https://doi.org/10.1093/nar/ gkq285

[30] WANG J, TANG Q, LU L, LUO Z, LI W et al. LncRNA OIP5-AS1 interacts with miR-363-3p to contribute to hepatocellular carcinoma progression through up-regulation of SOX4. Gene Ther 2020. https://doi.org/10.1038/s41434020-0123-2

[31] WEI F, YANG L, JIANG D, PAN M, TANG G et al. Long noncoding RNA DUXAP8 contributes to the progression of hepatocellular carcinoma via regulating miR-422a/ PDK2 axis. Cancer Med 2020; 9: 2480-2490. https://doi. org/10.1002/cam4.2861

[32] XIAO H. MiR-7-5p suppresses tumor metastasis of nonsmall cell lung cancer by targeting NOVA2. Cell Mol Biol Lett 2019; 24: 60. https://doi.org/10.1186/s11658-019-0188-3

[33] ZHENG Y, NIE P, XU S. Long noncoding RNA CASC21 exerts an oncogenic role in colorectal cancer through regulating miR-7-5p/YAP1 axis. Biomed Pharmacother 2020; 121: 109628. https://doi.org/10.1016/j.biopha.2019.109628

[34] PIAO HY, GUO S, WANG Y, ZHANG J. Long noncoding RNA NALT1-induced gastric cancer invasion and metastasis via NOTCH signaling pathway. World J Gastroenterol 2019; 25: 6508-6526. https://doi.org/10.3748/wjg.v25. i44.6508

[35] TANG LX, SU SF, WAN Q, HE P, XHANG Y et al. Novel long non-coding RNA LBX2-AS1 indicates poor prognosis and promotes cell proliferation and metastasis through Notch signaling in non-small cell lung cancer. Eur Rev Med Pharmacol Sci 2019; 23: 7419-7429. https://doi.org/10.26355/ eurrev_201909_18851

[36] SUN X, HUANG T, LIU Z, SUN M, LUO S. LncRNA SNHG7 contributes to tumorigenesis and progression in breast cancer by interacting with miR-34a through EMT initiation and the Notch-1 pathway. Eur J Pharmacol 2019; 856: 172407. https://doi.org/10.1016/j.ejphar.2019.172407 